\title{
A Dynamic Analysis of a Record Breaking Winter Season Blocking Event
}

\author{
Andrew D. Jensen \\ Department of Soil, Environmental, and Atmospheric Sciences, University of Missouri-Columbia, \\ 302 Anheuser Busch Natural Resources Building, Columbia, MO 65211, USA
}

Correspondence should be addressed to Andrew D. Jensen; jensenad@missouri.edu

Received 9 January 2015; Revised 21 March 2015; Accepted 13 April 2015

Academic Editor: Yafei Wang

Copyright (c) 2015 Andrew D. Jensen. This is an open access article distributed under the Creative Commons Attribution License, which permits unrestricted use, distribution, and reproduction in any medium, provided the original work is properly cited.

The objective of this work is to study in detail a strong North Pacific, large amplitude, and long-lived blocking event that occurred during January 23-February 16, 2014. Indeed, it was the 11th strongest Northern Hemisphere event lasting longer than 20 days since 1968. This event formed out of the strong ridge that was associated with the devastating drought in the Western United States during the winter season of 2013-2014. This blocking event had many outstanding dynamical characteristics, the chief of which was that it survived an abrupt change in the planetary-scale flow when the Pacific North American pattern index changed from positive to negative in early February. The block then reintensified and persisted into mid-February. Several diagnostic techniques are employed to investigate the change in the planetary-scale flow during early February 2014 that have been applied to blocking before but aren't as well known in the blocking literature.

\section{Introduction}

The classical picture of blocking anticyclones consists in a long lived (5 10 day minimum threshold of persistence), large amplitude, geopotential height anomaly [1-4]. More recently, some research groups have invoked the PV (potential vorticity) perspective in defining blocking (see, e.g., [5, 6]). Both methods define blocking as a significant persistent anomaly, positive height anomalies in the more classical framework, or negative PV anomalies, respectively. Some blocking events are characterized by height anomalies even more extreme than usual but are short lived; see [7]. Other blocking events are longer lived than others. However, longlived and larger than usual amplitude do not always coincide. For a blocking event to be both long-lived and large amplitude three conditions are typically fulfilled for North Atlantic events: (i) weak background westerly wind prior to block onset, (ii) strength of the storm track is strong leading up to the event, and (iii) large zonal scale wavenumbers [810]. As suggested in [9], however, these conditions likely prevail in North Pacific blocking events also. Furthermore, in [10] it was concluded that merger type blocking events were typically large amplitude compared to other blocking events. In this paper $I$ investigate the dynamics of a longlived, large amplitude, and merger type blocking event, which was associated with drought in the Western United States and also the cold temperatures in the Eastern part of the United States.

As explained in [11] blocking events are maintained by fluxes of anticyclonic vorticity into the blocking domain by synoptic-scale eddies. Moreover, several studies have examined the dynamics of blocking event life cycles by partitioning variables such as height or PV into synoptic, planetary, and interaction scales (with the interaction arising from nonlinearity in the PV tendency equation) [11, 12]. For example, in [13] North Atlantic blocking is shown to be planetary-scale dominant, while North Pacific blocking is more sensitive to transients on the synoptic-scale. As described in [11] Northern Hemisphere blocking events are maintained by nonlinear amplification between synoptic and planetary scales. Here, the blocking event under consideration was also maintained by nonlinear amplification in the manner described in [11]. Moreover, [11, 14] explain that blocking events may not survive an abrupt transition from one planetary-scale flow regime to another. The physical mechanism is likely due to a breakdown in the planetary-scale jet stream as it transitions to 
a new location or regime. The event under consideration here survived just such a regime change and various techniques will be used to examine the behavior of the event during the regime change and how nonlinear interactions sustained the event. Various techniques will be used to present evidence for the flow regime change.

The objective of this paper is to study in detail a North Pacific, large amplitude as quantified by the block intensity index (BI) (to be explained below), and long-lived blocking event that occurred during January 23-February 16, 2014, centered at $130^{\circ} \mathrm{W}$. Its association with the devastating drought in California alone warrants further study of the event. However, as will be shown it had many outstanding dynamical characteristics as well, the chief of which is that it survived an abrupt change in the planetary-scale flow when the Pacific North America (PNA) pattern index changed from positive to negative in early February. The event then reintensified, thus increasing its longevity. This event is also part of the drought associated ridge over the West Coast of the United States during the 2013-2014 winter that has been shown to have an anthropogenic footprint; see [15]. In this paper several diagnostic techniques will be employed and another objective of this work is to illustrate techniques that have recently been applied to blocking but aren't as well known in the literature on blocking. In Section 2, the various techniques used to study the event are presented in some detail. Section 3 contains a general analysis of the planetaryscale environment out of which the event formed and the main results of the paper. The paper ends in Section 4 with a discussion of the results.

\section{Data and Methods}

2.1. Data. The data set used to examine the dynamics of this event was the National Centers for Environmental Prediction/National Center for Atmospheric Research reanalyses of the standard atmospheric variables: sea level pressure, zonal and meridional winds, geopotential height, and temperature. These variables were used to calculate the wave activity flux, integrated enstrophy, as well as the PV, and PV tendency on the $315 \mathrm{~K}$ surface.

2.2. Methods and Diagnostics. A more complete description of the blocking index used in this study can be found in [7]; however, it can be described as integrating both the subjective Rex criteria (see $[1,2]$ ) and the objective Lejenas-Okland criteria (see [3]) but with a minimum threshold of persistence of five days. This index was used to detect the blocking onset and decay times for the event considered in this study.

The PV was calculated on the $315 \mathrm{~K}$ surface:

$$
\mathrm{PV}=\rho^{-1} \zeta_{a} \cdot \nabla \theta
$$

where $\zeta_{a}$ is absolute vorticity and $\theta$ is the potential temperature. The change in block center point PV was calculated using the method outlined in [11]. As described there, the development of a blocking event is described by the advection of PV:

$$
\frac{\partial \mathrm{PV}}{\partial t}=-\mathbf{v}_{h} \cdot \nabla \mathrm{PV}
$$

The respective roles of the synoptic-scale and planetaryscale forcings may be a examined by the methodology of [11, 12]. The scales were obtained by substituting $\mathrm{PV}=\overline{\mathrm{PV}}+\mathrm{PV}^{\prime}$ into the above tendency equation where the overbar (prime) denotes the planetary-scale (synoptic-scale) component to get

$$
\frac{\partial \mathrm{PV}}{\partial t}=\left.\frac{\partial \mathrm{PV}}{\partial t}\right|_{P}+\left.\frac{\partial \mathrm{PV}}{\partial t}\right|_{S}+\left.\frac{\partial \mathrm{PV}}{\partial t}\right|_{I}=P+S+I,
$$

where $P, S$, and $I$, represent the planetary, synoptic, and scale interaction PV advections, respectively, where $I$ represents nonlinear interactions. To separate the planetary-scale wavelengths from the synoptic-scale wavelengths a second order Shuman filter was used.

The $500 \mathrm{hPa}$ geopotential heights $Z$ were also filtered with a Shuman filter to retain the planetary-scale waves $(\geq 6000 \mathrm{~km})$. The synoptic-scale heights were calculated as a residual: $Z_{s}=Z-Z_{p}$. Following the method of [16] the planetary-scale height field was averaged over a $40^{\circ}$ latitude by $60^{\circ}$ longitude box encompassing the block domain. Plots of $Z_{p}$ and $Z_{s}$ were then used to determine the scale dominance of the blocking event: planetary-scale, synoptic-scale, and alternating, where, as explained in [16] these represent three classes of solutions of the nonlinear barotropic vorticity equation. The heights were compared to their monthly averages to determine scale dominance. If the planetary-scale (synoptic-scale) heights in the block domain are not greater than the monthly average of the planetaryscale (synoptic-scale) heights throughout the duration of the block, the event is classified as alternating.

As noted in [17] the planetary-scale flow in midlatitudes has cyclic regimes. These regimes arise from the propagation and dispersion of Rossby waves. Phase diagrams can be used to effectively display these cyclic regimes [17]. To determine the evolution dynamics of block formation and maintenance, trajectories of a variable $X$ can be plotted in the phase plane, where the horizontal coordinate is $X$ and the vertical coordinate is $d X / d t$. In this study the trajectories of $X=$ geopotential height were analyzed by calculating $d X / d t$ with 4 th-order finite differencing truncation in a $40^{\circ}$ by $60^{\circ}$ box for the duration of the blocking event. This method describes the time-evolution in phase space of the blocking event as a dynamical system. In the ideal autonomous case, for cyclic regimes, the trajectories correspond to a harmonic oscillator and are circular. For stable regimes, trajectories approach a limit cycle. For this reason, the phase diagram is suitable to determine regime changes. Since the atmosphere is nonautonomous the attractor regime may change its character and the trajectories may cross indicating explicit time-dependence of the governing equations.

A series of recent studies (see [18] and the references therein) have demonstrated that enstrophy may be used as an indicator of the stability of large-scale flows. Enstrophy based 


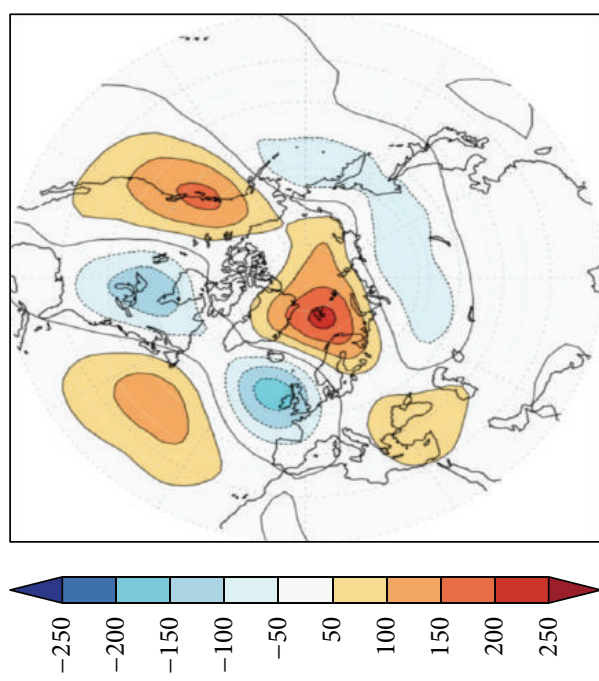

(a)

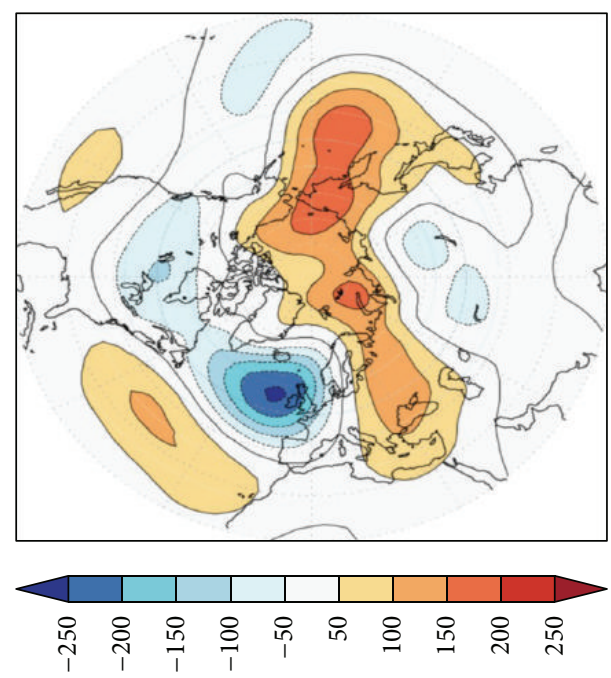

(b)

FIGURE 1: (a) January 2014 geopotential height anomalies in (m). (b) February 2014 geopotential height anomalies.

diagnostics have been used to identify the change in flow regimes, especially the onset and decay periods for blocking. The diagnostic used here is the domain integrated enstrophy (IRE):

$$
\operatorname{IRE} \equiv \int_{A} \zeta^{2} \mathrm{~d} A,
$$

where the area $A$ over which the integral is evaluated can be an entire hemisphere, or the blocking domain, such as a 40 by 60 degree box around the blocking event [16]. Relative maxima in the IRE time series in the blocking domain under consideration represent increased instability and correlate well with block onset and decay, while during the maintenance phase of a block, the IRE dips to a minimum. The instability indicated by the IRE is correlated with the sum of the positive finite-time Lyapunov exponents [19].

\section{Synoptic and Dynamic Analysis}

3.1. Synoptic- and Planetary-Scale Analysis. The event considered here occurred during January 23-February 16, 2014 and was centered at $130^{\circ} \mathrm{W}$. This blocking event developed from an anomalously strong ridge over the west coast of the United States. In this section the environment in which the block formed and was maintained will be discussed.

In January (for details see NCDC, State of the climate: Synoptic discussion for January 2014, http://www.ncdc .noaa.gov/sotc/synoptic/2014/1) positive SST anomalies grew more extensive in the North Pacific, while the East PacificNorth Pacific pattern index (EP-NP) was positive. The Pacific North American pattern index was also positive during January. However, the circulation and temperature patterns during January resembled the EP-NP pattern more than the PNA pattern as noted by the NCDC and can be seen in the geopotential height and temperature fields (not shown). Both the SSTs and the circulation pattern contributed to the ridging over the west coast from which the blocking event formed.
February was somewhat different (for details see NCDC, State of the climate: Synoptic discussion for January 2014, http://www.ncdc.noaa.gov/sotc/synoptic/2014/2). The NCDC notes that there was no teleconnection pattern that captured the circulation completely. However, the EP-NP pattern continued positive, while, the PNA pattern changed from positive to negative in the first part of the month, indicating that there was a change in the flow regime. This can be seen in Figure 1, where a clear shift in the alternating pattern of positive/negative height anomalies from January has occurred; that is, the jet has shifted. As noted in the introduction, blocking events are expected to decay in the event of such a change. However, as will be shown in the next subsection, the blocking event was maintained in spite of the large-scale flow regime change. Additional evidence for the change will be presented in the next section. Also, the SSTs in the blocking region become anomalously strong during the early part of February (see Figure 2), likely contributing to the maintenance of the blocking event.

This blocking event that formed under the influence of the described conditions was a long-lived and large amplitude event. According to the blocking event archive at http://solberg.snr.missouri.edu/gcc/ it is the 11th strongest Northern Hemisphere event lasting longer than 20 days since 1968 as measured by its Block Intensity index (BI) of 5.93. Block intensity is the dimensionless quantity defined as

$$
\mathrm{BI}=100\left[\left(\frac{Z_{\max }}{Z}\right)-1\right] \text {. }
$$

Here $Z_{\max }$ is the maximum $500 \mathrm{hPa}$ height in the closed anticyclone region or on a line associated with the ridge and $Z$ is the subjectively chosen $500 \mathrm{hPa}$ height contour encompassing the upstream and downstream troughs; see [7] for a more thorough explanation. The BI measures the amplitude of the quasi-stationary wave in the blocking region. The BI was calculated daily during the event; see Figure 3. 


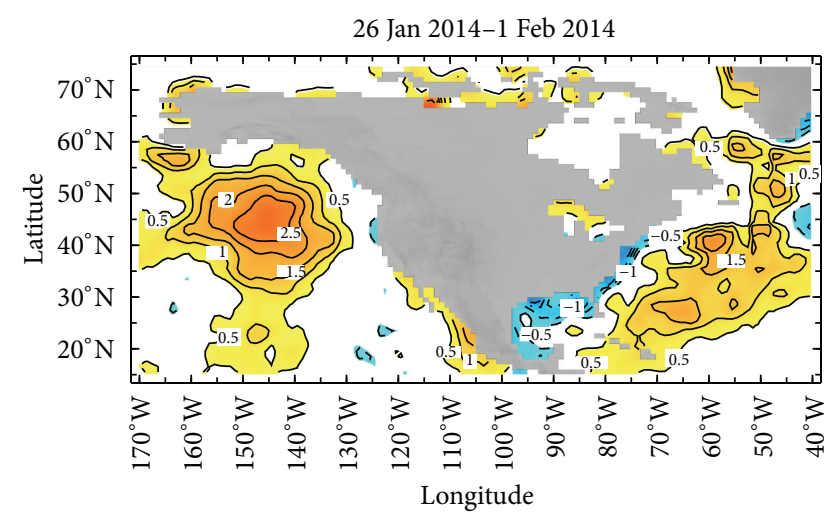

(a)

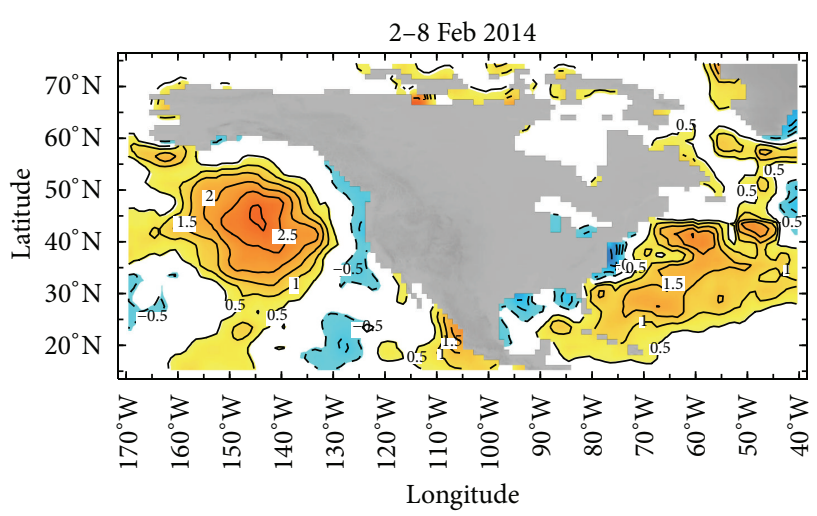

(b)

Figure 2: (a) SST anomalies 26 Jan 2014-1 Feb 2014. (b) SST anomalies 2-8 Feb 2014.

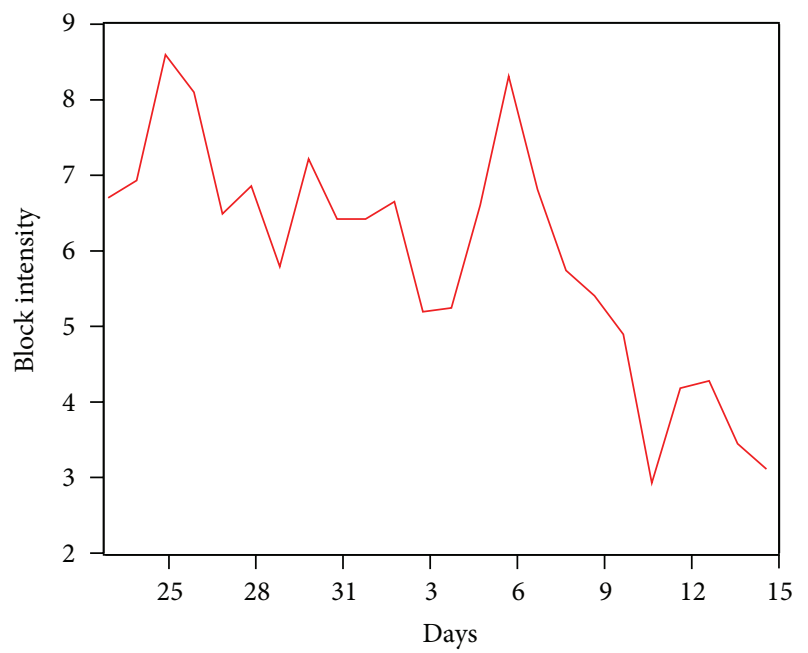

FIGURE 3: Block intensity (dimensionless) as a function of time: January 21-February 15, 2014.

TABLE 1: Time periods of the blocking event partitioned using the BI.

\begin{tabular}{lc}
\hline Phase & Time period \\
\hline Onset & January 21-22 \\
Intensification & January 23-25 \\
Maintenance 1 & January 26-February 2 \\
Intensification 2 & February 3-6 \\
Maintenance 2 & February 7-10 \\
Intensification 3 & February 11-13 \\
Decay & February 14-16 \\
\hline
\end{tabular}

The BI was used to partition the blocking event into different phases, as in [11]; see Table 1, which will be used below in the PV tendency calculations.

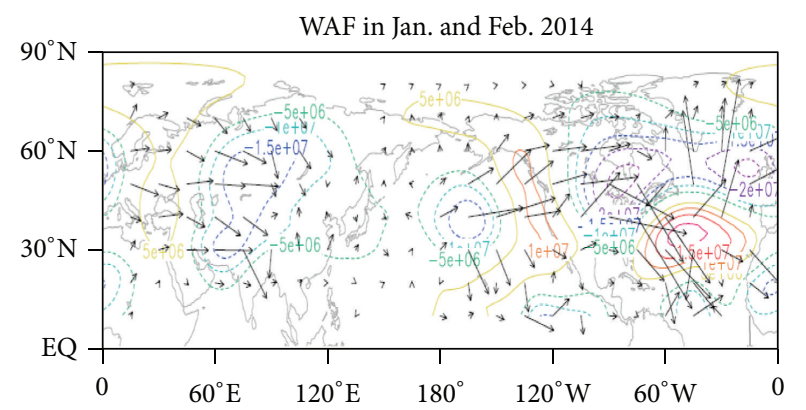

FIgURE 4: Rossby Wave Activity Flux in January and February 2014.

\subsection{Dynamic Analysis}

(a) General Description of the Event. As described in [15], the ridge out of which the blocking event originated was in part formed by wave-activity fluxes from the subtropical Pacific. The formation of the ridge over the west coast of the United States was examined using the Rossby wave activityflux, defined by Takaya and Nakamura [20] for the prediction of propagating planetary waves in mean flows. Additionally, [20] demonstrates that the absorption of Rossby wave packets is instrumental in the formation of blocking. As can be seen in Figure 4, a persistent source of Rossby wave energy was absorbed in the Gulf of Alaska in January-February throughout the event thus sustaining it. Moreover, Rossby wave energy originated there and propagated to the eastern US possibly contributing to the extreme cold temperatures there [15].

This event followed the [4] Tsou and Smith conceptual model in its formation, which specifies that a blocking event forms when the following ingredients are in phase: (i) a planetary-scale quasi-stationary $500 \mathrm{hPa}$ ridge, (ii) a developing precursor upstream surface cyclone, (iii) an associated amplifying $500 \mathrm{hPa}$ upstream short-wave ridge, and (iv) strong jet maximum.

As described above, a strong quasi-stationary planetaryscale ridge over the West Coast of the United States was present; see [15] for a more thorough description. 


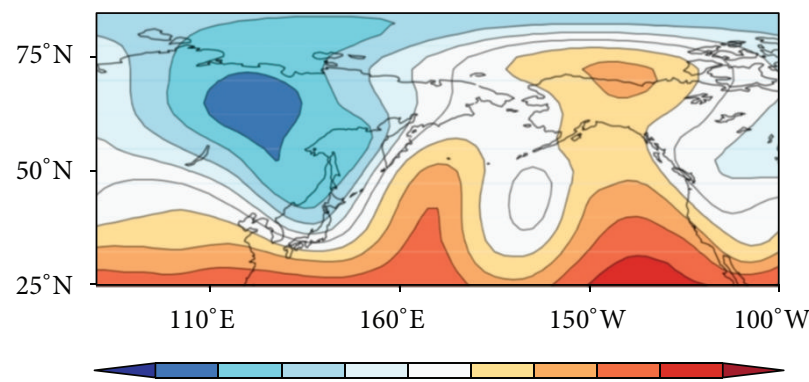

4900500051005200530055005600570058005900

(a)

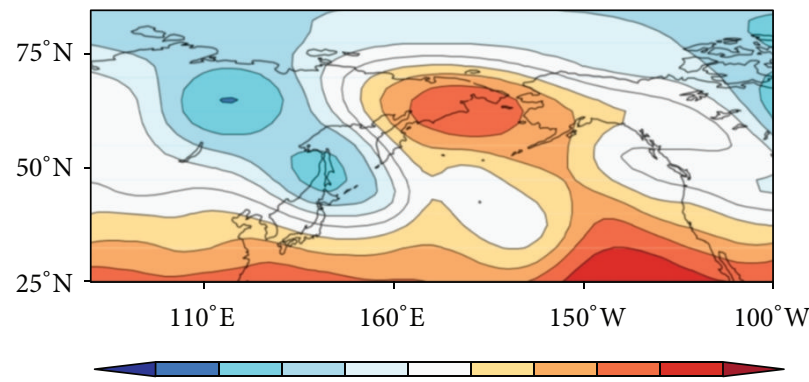

4900500051005200530055005600570058005900

(c)

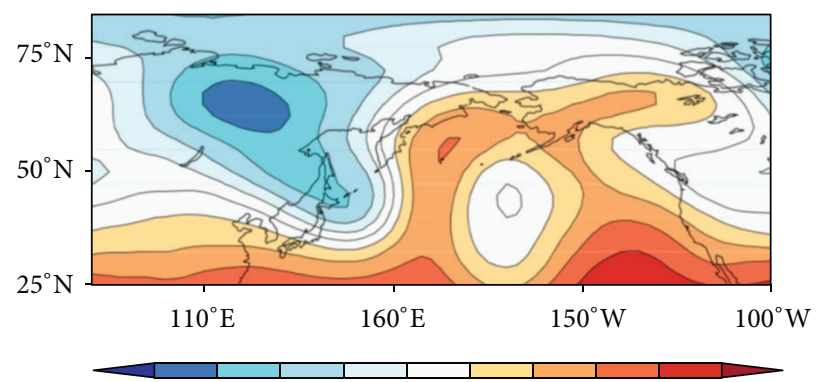

4900500051005200530055005600570058005900

(b)

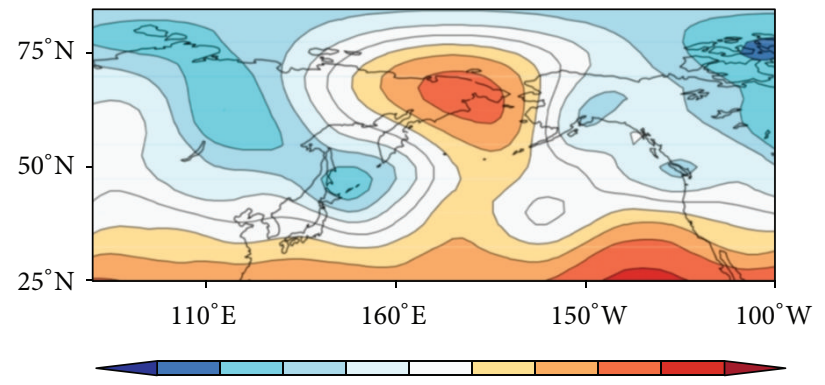

4950505051505250535055505650575058505950

(d)

Figure 5: Upstream $500 \mathrm{hPa}$ ridge in (m) merging with the block: (a) Feb. 4th, (b) Feb. 5th, (c) Feb. 6th, and (d) Feb. 7th.

As described in [4], blocking events are preceded by upstream surface cyclogenesis. In this event, there was a deep (966 mb at one point) cyclone that abutted the ridge by the 20th of January, three days before the ridge became a blocking event. Along with that there was an amplifying $500 \mathrm{hPa}$ upstream short-wave ridge that can be described by processes explained below. Finally, the strong jet maximum imparted anticyclonic vorticity to the blocking region.

By the 4th of February a strong positive $500 \mathrm{hPa}$ height anomaly (long-wave ridge) was building in the Western Pacific (see Figure 5), while the original event was moving westward. By the 6th these two ridges merge to restrengthen the blocking event. This merger was accompanied by significant positive SST anomalies as well (see Figure 2). Finally, the event dissipated as strong cyclogenesis entered the region and an upper level trough finally eroded the blocking ridge by mid-February.

(b) Long-Lived and Large Amplitude Criteria. As described in the Introduction and in [10] long-lived and large-amplitude blocking events typically have (i) weak background westerly wind before and at block onset, (ii) measures of storm track strength should indicate a strong storm track before onset, and (iii) large zonal scale wavenumbers. To determine if the background winds were weak at the time before and at block formation for this event the zonal winds were averaged for three weeks prior to the event and then the two-year average was subtracted. As can be seen in Figure 6, the background westerlies were indeed weak upstream of the blocking event providing a favorable environment for a long-lived and largeamplitude event. Next, a measure of storm track strength was

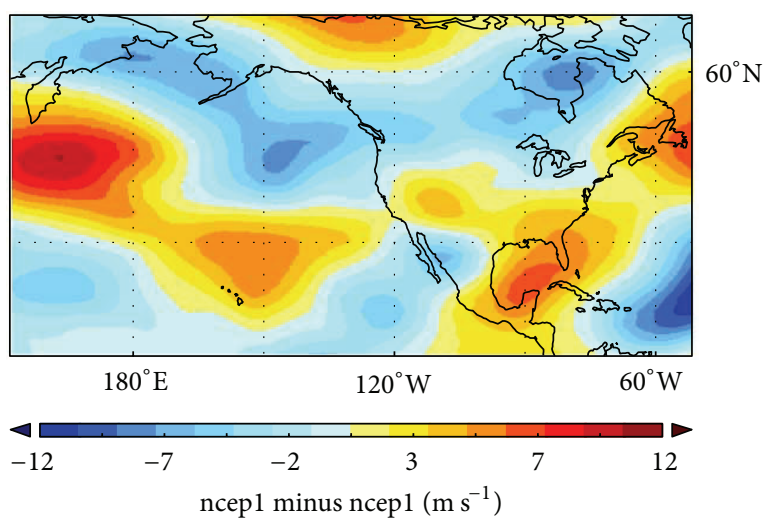

FIGURE 6: Averaged westerly winds in Jan 2014 minus averaged westerly winds 2012-2014.

computed. As outlined in [21], the 300-hPa meridional wind variance $V_{1 d f}$, with a 24-hour difference filter, may be used to measure the storm track strength:

$$
V_{1 d f}=\overline{\left[V_{(t+24 \mathrm{~h})}-V_{(t)}\right]^{2}} .
$$

The overbar denotes a time-average. As described in [21] this measure is comparable to other measures of storm track strength. Contours for $V_{1 d f}$ were plotted (see Figure 7) for January 2014 and for Nov-Dec 2013. As can be seen in Figure 7, the storm track strength increased prior to the blocking event.

(c) Height Scale-Partitioning. The method used to filter and partition the geopotential heights into planetary- and 


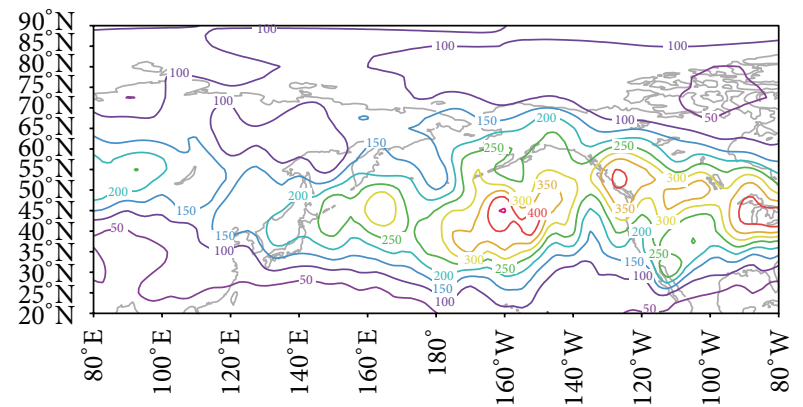

(a)

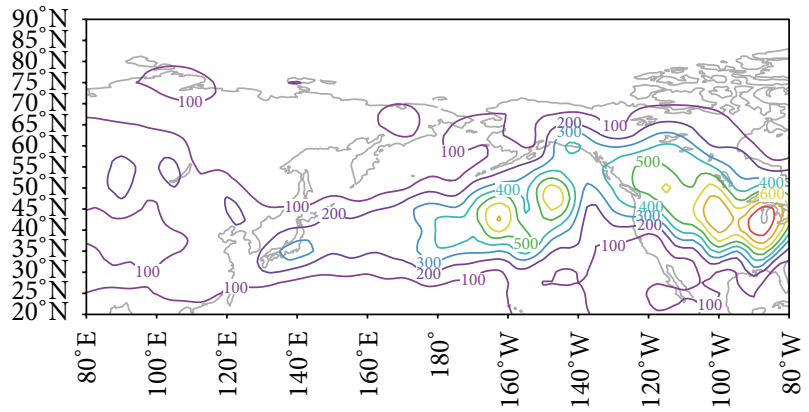

(b)

FIgURE 7: (a) $V_{1 d f}$ for Nov-Dec 2013. (b) $V_{1 d f}$ for 1-23 Jan 2014. Units: $\left(\mathrm{m}^{2} \mathrm{~s}^{-2}\right)$.

synoptic-scales was reviewed in Section 2.2. The scale partitioned heights were compared to their monthly averages in January and February as in [16]. The planetary-scale heights (see Figure 8) are above their monthly average in the blocking domain until approximately February 7 th and then remain below the average for the rest of the duration of the blocking event, indicating that planetary-scale processes may have had a significant role in the preconditioning and onset dynamics of the event. Figure 8 also shows the loss of support by the planetary-scale that led to the decay of the event. The synoptic-scale heights are initially above their monthly average (corresponding to the amplifying $500 \mathrm{hPa}$ short-wave ridge in the Tsou and Smith model) but soon decrease below the average. Near February 7th the synopticscale heights begin to rise near the average and soon exceed it (see Figure 9) before finally dipping below the average at block decay. This event can be classified as an alternating scale event as in [16] since neither the planetary nor synoptic scale was above its monthly average throughout the duration of the event. This indicates that both scales play important roles in the formation (both contribute positively) and maintenance of the event (planetary-scale loses support, synoptic-scale contributes positively). The loss of support of the planetaryscale and the gain in support of the synoptic-scale around February 7 th coincide with a marked increase in the BI (see Figures 3 and 8, Section 3.1).

(d) Phase Diagram. The method of calculation and use of phase diagrams were explained briefly in Section 2.2. For the phase diagram used here trajectories of planetary-scale heights during the blocking event were plotted; see Figure 10: $Z_{p}$ (planetary-scale height) versus $d Z_{p} / d t$ in a $40^{\circ}$ by $60^{\circ}$ box. The trajectory in the blocking domain quickly goes into a limit cycle, representing a stable regime. Around February 67 the trajectory leaves the first limit cycle which corresponds to a change in planetary-scale flow regime. The trajectory goes into another small limit cycle before the event finally decays.

(e) IRE. As described in Section 2.2, enstrophy and enstrophy based relationships can be used as indicators of the stability of large-scale flows. These quantities can be used to identify

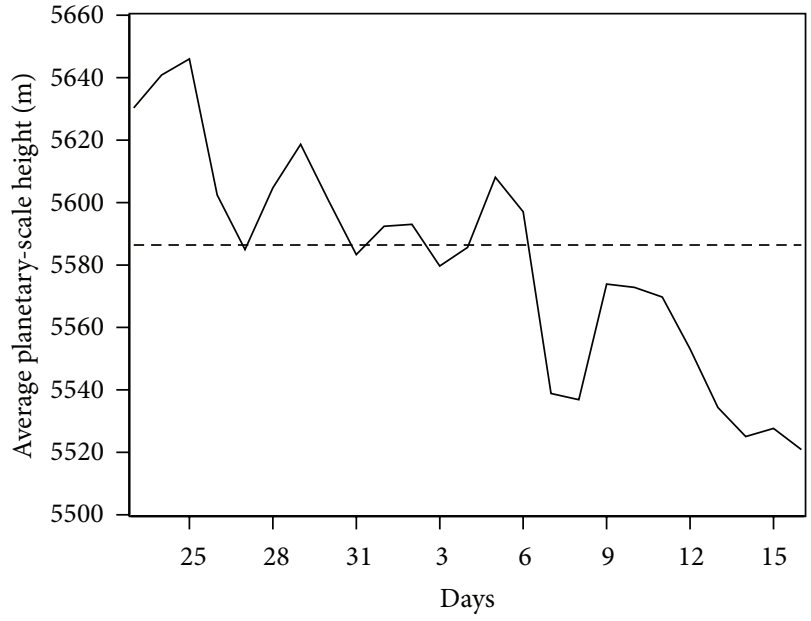

Figure 8: Planetary-scale $500 \mathrm{hPa}$ geopotential heights (m) for January 23-February 16 . The horizontal line represents monthly mean.

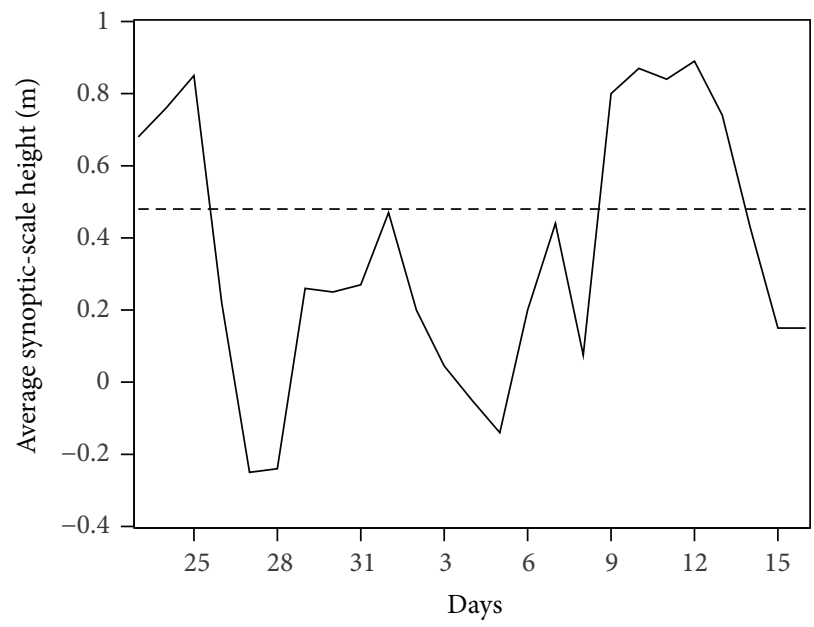

FIgURE 9: Synoptic-scale $500 \mathrm{hPa}$ geopotential heights $(\mathrm{m})$ for January 23-February 16 . The horizontal line represents monthly mean. 


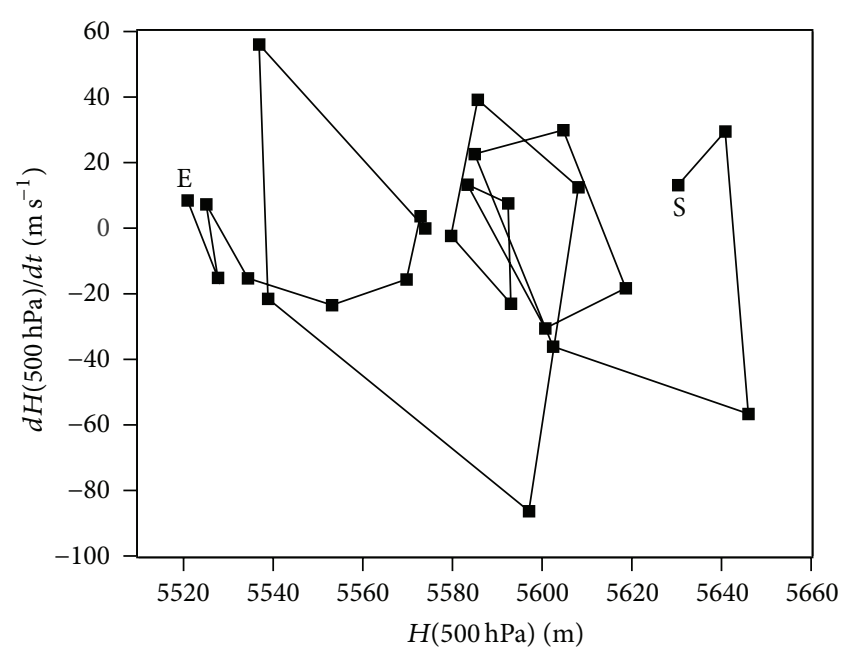

FIGURE 10: Phase diagram of $500 \mathrm{hPa}$ planetary-scale heights for January 23-February 16. Each point represents a day. $S=$ start and E $=$ end.

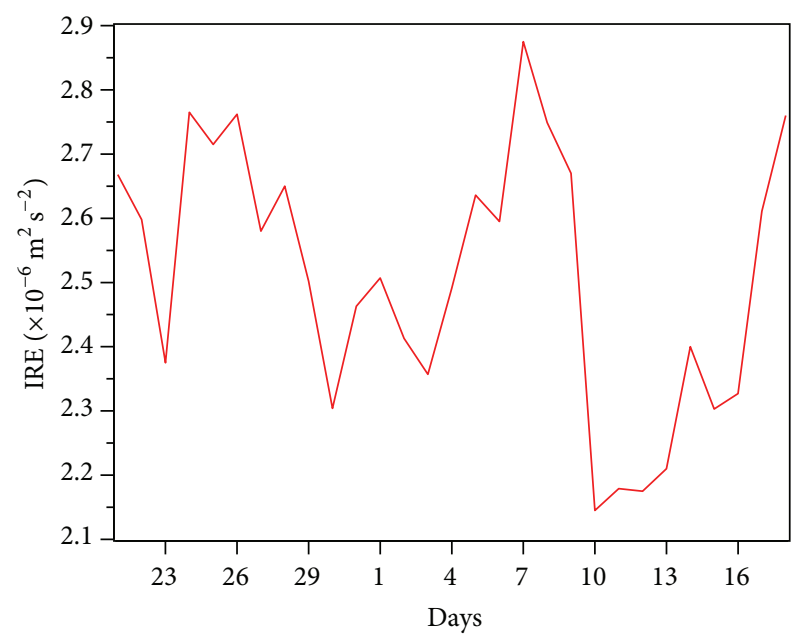

FIGURE 11: Integrated enstrophy (IRE) for January 21-February 18.

the change in flow regimes, including the onset and decay periods for blocking events. The past research has shown that block onset and decay are associated with relative maxima in the enstrophy time series. Typically, during the maintenance phase of a blocking event, the enstrophy dips to a relative minimum. The IRE time series for this event is shown in Figure 11. The IRE reaches a relative maximum near onset and rises to another relative maximum at block decay, indicating increased instability as described in Section 2.2. The IRE then dips to a relative minimum. However, near February 7th, the IRE rises to a significant maximum, indicating a change in the planetary-scale dynamics. This, along with the shift in the pattern of high/low in the height field which can be seen in Figures 1 and 5 and in the PNA pattern index change from

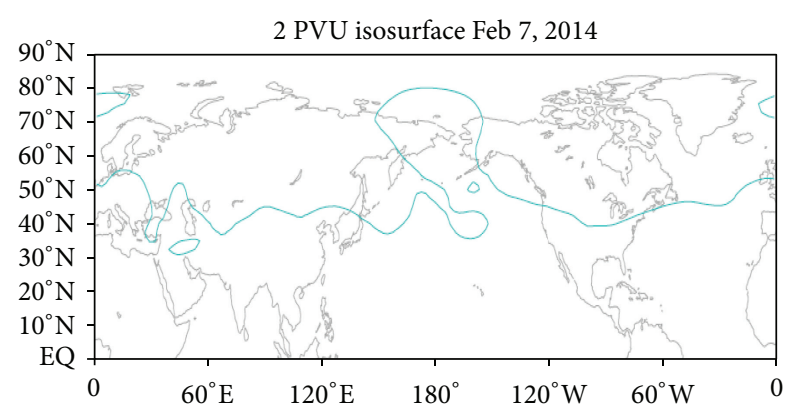

FIgURE 12: 2 PVU surface for February 7, 2014.

positive to negative, lends evidence to the change in the flow regime.

(f) $P V$. The PV tendency was calculated as explained in Section 2.2. The filtered PV tendencies were calculated at the blocking center point and time averaged for specific time periods during the blocking event; see Tables 1 and 2. The synoptic-scale tendency is mainly supportive of negative PV advection in the blocking region except at onset and decay, while the interaction PV alternates signs. The planetary-scale $\mathrm{PV}$ is mostly positive, suggesting that it does not have a significant role in maintaining the block. However, during the Maintenance period 2 (see Tables 1 and 2), all three scales play a significant role in the maintenance of the blocking event, implying that the interaction between scales is nonlinear (see [11]). As discussed above, the blocking ridge merges with another developing ridge during this time. Also, as seen in Figure 12 the cyclonic PV streamer may have played a role in sustaining the block by the diabatic depletion of PV at upper levels [22]. The location of the cyclonic PV streamer corresponds with the trough to the east seen in Figure 5. There is cyclogenesis in the central Pacific between the blocking ridge and the developing ridge in the western Pacific, as represented in the figure by the cyclonic PV streamer, which eventually becomes the eastern part of the omega block.

\section{Discussion and Conclusions}

This long-lived event (January 23-February 16, 2014) formed out of the ridge over the West Coast of the United States associated with severe drought conditions in California that has been shown to have an anthropogenic footprint; see [15]. The EP-NP pattern index was positive, indicating there was a favorable environment for the block to form. Moreover, as explained in [15] and as seen in Figure 4, Rossby wave energy dispersed downstream of the event which deepened the trough over the northeastern part of North America. This led to plunging temperatures and the "polar vortex." Additionally, this event was the 11th strongest of the events that lasted longer than 20 days; see http://solberg.snr.missouri.edu/gcc/.

Several of the techniques used here detail the evidence of a large-scale change in the dynamics seen in Figure 1, which was essentially a nonlinear change. The phase diagram, IRE, scale-partitioned heights, and PV all show changes in 
the dynamics in early February. The phase diagram shows the change from a more stable state to a more unstable state in early February. The IRE reflects the instability during this time period as well. The scale-interaction PV demonstrates that the change in dynamics was essentially nonlinear by the scale interaction between planetary and synoptic scales. The scale-partitioned eddy heights also increase abruptly in early February at the time the blocking amplitude (BI) increases; see Figure 3.

One of the most significant aspects of this event is that it was long-lived and large-amplitude in the face of regime change. Several of the techniques also detail the evidence for the event to be both long-lived and large-amplitude. As detailed in [10] the weak background westerlies prior to block onset and the significant strength of the storm track prior to onset also provide favorable conditions for long-lived and large-amplitude blocking events. The event under consideration here was long-lived and large-amplitude and had weak background westerlies and large storm track strength prior to block onset (see Figures 6 and 7 and Section 3.2). The IRE (integral of enstrophy) in wavenumber space represents energy dispersion and can also be seen as a proxy for storm track strength as it increased to a relative maximum before block onset. The positive change in SSTs in the blocking region during the regime change likely helped sustaining the blocking event as well. As described in [11] fluxes of low PV air into the blocking region maintain blocking events. Also, as seen in Figure 12 the cyclonic PV streamer may have played a role in sustaining the block by the diabatic depletion of PV at upper levels [22]. Moreover, the PV scale-interaction demonstrates that the regime change was nonlinear in nature leading to nonlinear amplification as can be seen in the eddy heights as their increase coincides with the blocking amplitude increase (see Figures 3 and 9).

Several techniques were employed in the study of the dynamics of this event. Some of the techniques used here are not as well known in the literature on blocking but are effective at illustrating the exceptional nature of this event as it survived a regime change. A potential weakness in all of the techniques employed here is that they tend to emphasize the $500 \mathrm{hPa}$ surface dynamics, somewhat arbitrarily; see [6]. Also, the temperature advection and other thermodynamic variables are not taken into account either. The work here also does not use the blocking eddy-interaction method as in [8], as it is outside the scope of this work. However, the techniques used here give a reasonably comprehensive view of the dynamics of the event, especially the changing nature of the flow in early February.

The suggestion in $[11,14]$ that blocking events may not survive an abrupt transition from one planetary-scale flow regime to another is here shown to be not entirely correct. The event under consideration here was a strong event that survived a large-scale change in flow regimes as evidenced by the changes in PNA pattern index, the height pattern as seen in Figure 1, the phase diagram, and the IRE. The nonlinear interaction as evidenced by the PV in Table 2 and the merger between the two ridges shown in Figures 5 and 9 sustained the blocking event in the face of potential dissipation. Thus, fortuitous synergistic nonlinear interaction
TABLE 2: Scale partitioned PV tendency on $315 \mathrm{~K}$ Surface: PV $\times$ PVU day $^{-1}, 1$ PVU $\equiv 10^{-6} \mathrm{Km}^{2} \mathrm{~kg}^{-1} \mathrm{~s}^{-1}$.

\begin{tabular}{lccc}
\hline Phase & $\begin{array}{c}\text { Planetary-scale } \\
\text { PV }\end{array}$ & $\begin{array}{c}\text { Synoptic-scale } \\
\text { PV }\end{array}$ & $\begin{array}{c}\text { Interaction } \\
\text { PV }\end{array}$ \\
\hline Onset & 1.06 & 0.008 & 0.06 \\
Intensification & 0.82 & -0.018 & -0.028 \\
Maintenance 1 & 3.005 & -0.044 & -0.99 \\
Intensification 2 & 0.82 & -0.048 & 0.39 \\
Maintenance 2 & -2.2 & -0.058 & -0.98 \\
Intensification 3 & 9.62 & -0.17 & 0.48 \\
Decay & 13.024 & 0.019 & 5.13 \\
\hline
\end{tabular}

may have increased the persistence in the face of regime change.

\section{Symbols and Acronyms}

$\begin{array}{ll}\text { BI : } & \text { Block intensity index } \\ \text { EP-NP: } & \text { East Pacific-North Pacific pattern } \\ I: & \text { Interaction-scale PV } \\ \text { IRE: } & \text { Integrated enstrophy } \\ P: & \text { Planetary-scale PV } \\ \text { PNA: } & \text { Pacific North America pattern } \\ \text { PV: } & \text { Potential vorticity } \\ S: & \text { Synoptic-scale PV } \\ \text { SST: } & \text { Sea surface temperature } \\ V_{1 d f}: & \text { 300-hPa meridional wind variance } \\ Z_{\text {max }}: & \text { Maximum 500 hPa height in closed height } \\ & \text { contour of the block } \\ Z_{p}: & \text { Planetary-scale geopotential height } \\ Z_{s}: & \text { Synoptic-scale geopotential height. }\end{array}$

\section{Conflict of Interests}

The author declares that there is no conflict of interests regarding the publication of this paper.

\section{Acknowledgments}

The author wishes to thank the anonymous reviewers for their insightful and helpful comments, which have considerably improved the clarity and strength of this paper. The author also wishes to thank Dr. Anthony Lupo for insightful discussions regarding this work.

\section{References}

[1] D. F. Rex, "Blocking action in the middle troposphere and its effect upon regional climate," Tellus, vol. 2, no. 3, pp. 196-211, 1950.

[2] D. F. Rex, "Blocking action in the middle troposphere and its effect upon regional climate," Tellus, vol. 2, no. 4, pp. 275-301, 1950.

[3] H. Lejenas and H. Okland, "Characteristics of Northern Hemisphere blocking as determined from a long time series of observational data," Tellus A, vol. 35, no. 5, pp. 350-362, 1983. 
[4] C. H. Tsou and P. J. Smith, "The role of synoptic/planetary-scale interactions during the development of a blocking anticyclone," Tellus, vol. 42, no. 1, pp. 174-193, 1990.

[5] C. Schwierz, M. Croci-Maspoli, and H. C. Davies, "Perspicacious indicators of atmospheric blocking," Geophysical Research Letters, vol. 31, no. 6, Article ID L06125, 2004.

[6] D. Small, E. Atallah, and J. R. Gyakum, "An objectively determined blocking index and its Northern Hemisphere climatology," Journal of Climate, vol. 27, no. 8, pp. 2948-2970, 2014.

[7] J. M. Wiedenmann, A. R. Lupo, I. I. Mokhov, and E. A. Tikhonova, "The climatology of blocking anticyclones for the Northern and Southern Hemispheres: block intensity as a diagnostic," Journal of Climate, vol. 15, no. 23, pp. 3459-3473, 2002.

[8] D. Luo, "A barotropic envelope Rossby soliton model for BlockEddy interaction. Part I: effect of topography, Journal of the Atmospheric Sciences, vol. 62, no. 1, pp. 5-21, 2005.

[9] D. Luo, A. R. Lupo, and H. Wan, "Dynamics of eddy-driven low-frequency dipole modes. Part I: a simple model of North Atlantic Oscillations," Journal of the Atmospheric Sciences, vol. 64, no. 1, pp. 3-28, 2007.

[10] D. Luo, J. Cha, L. Zhong, and A. Dai, "A nonlinear multiscale interaction model for atmospheric blocking: the eddy-blocking matching mechanism," Quarterly Journal of the Royal Meteorological Society, vol. 140, pp. 1785-1808, 2014.

[11] J. P. Burkhardt and A. R. Lupo, "The planetary- and synopticscale interactions in a southeast Pacific blocking episode using PV diagnostics," Journal of the Atmospheric Sciences, vol. 62, no. 6, pp. 1901-1916, 2005.

[12] S. J. Colucci, "Planetary-scale preconditioning for the onset of blocking," Journal of the Atmospheric Sciences, vol. 44, pp. 124139, 2001.

[13] H. Nakamura, M. Nakamura, and J. L. Anderson, "The role of high- and low-frequency dynamics in blocking formation," Monthly Weather Review, vol. 125, no. 9, pp. 2074-2093, 1997.

[14] K. Haines and A. J. Holland, "Vacillation cycles and blocking in a channel," Quarterly Journal of the Royal Meteorological Society, vol. 124, pp. 873-897, 1998.

[15] S. Y. Wang, L. Hipps, R. R. Gillies, and J. H. Yoon, "Probable causes of the abnormal ridge accompanying the 2013-2014 California drought: ENSO precursor and anthropogenic warming footprint," Geophysical Research Letters, vol. 41, no. 9, pp. 32203226, 2014.

[16] H. Athar and A. R. Lupo, "Scale and stability analysis of blocking events from 2002-2004: a case study of an unusually persistent blocking event leading to a heat wave in the Gulf of Alaska during August 2004," Advances in Meteorology, vol. 2010, Article ID 610263, 15 pages, 2010.

[17] A. R. Lupo, I. I. Mokhov, S. Dostoglou, A. R. Kunz, and J. P. Burkhardt, "Assessment of the impact of the planetary scale on the decay of blocking and the use of phase diagrams and enstrophy as a diagnostic," Izvestiya, Atmospheric and Ocean Physics, vol. 43, no. 1, pp. 45-51, 2007.

[18] A. D. Jensen and A. R. Lupo, "Using enstrophy as a diagnostic to identify blocking regime transition," Quarterly Journal of the Royal Meteorological Society, vol. 682, pp. 1677-1683, 2014.

[19] V. P. Dymnikov, Y. V. Kazantsev, and V. V. Kharin, "Information entropy and local Lyapunov exponents of barotropic atmospheric circulation," Izvestiya, Atmospheric and Oceanic Physics, vol. 28, no. 6, pp. 425-432, 1992.
[20] K. Takaya and H. Nakamura, "A formulation of a phaseindependent wave-activity flux for stationary and migratory quasigeostrophic eddies on a zonally varying basic flow," Journal of the Atmospheric Sciences, vol. 58, no. 6, pp. 608-627, 2001.

[21] N. Harnik and E. K. M. Chang, "Storm track variations as seen in radiosonde observations and reanalysis data," Journal of Climate, vol. 16, no. 3, pp. 480-495, 2003.

[22] R. W. Moore, O. Martius, and T. Spengler, "The modulation of the subtropical and extratropical atmosphere in the Pacific basin in response to the Madden-Julian oscillation," Monthly Weather Review, vol. 138, no. 7, pp. 2761-2779, 2010. 

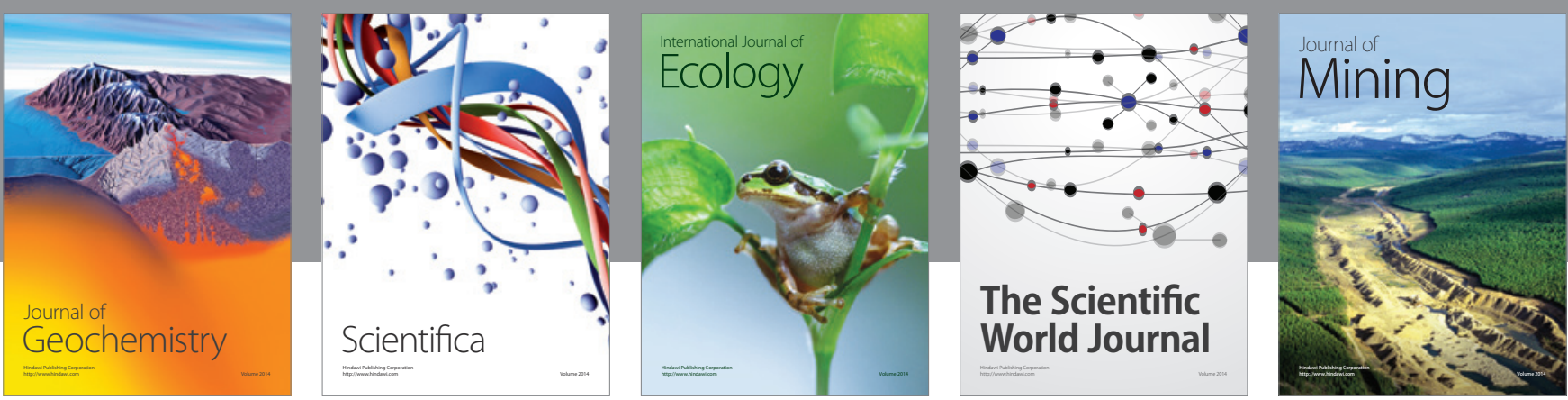

The Scientific World Journal
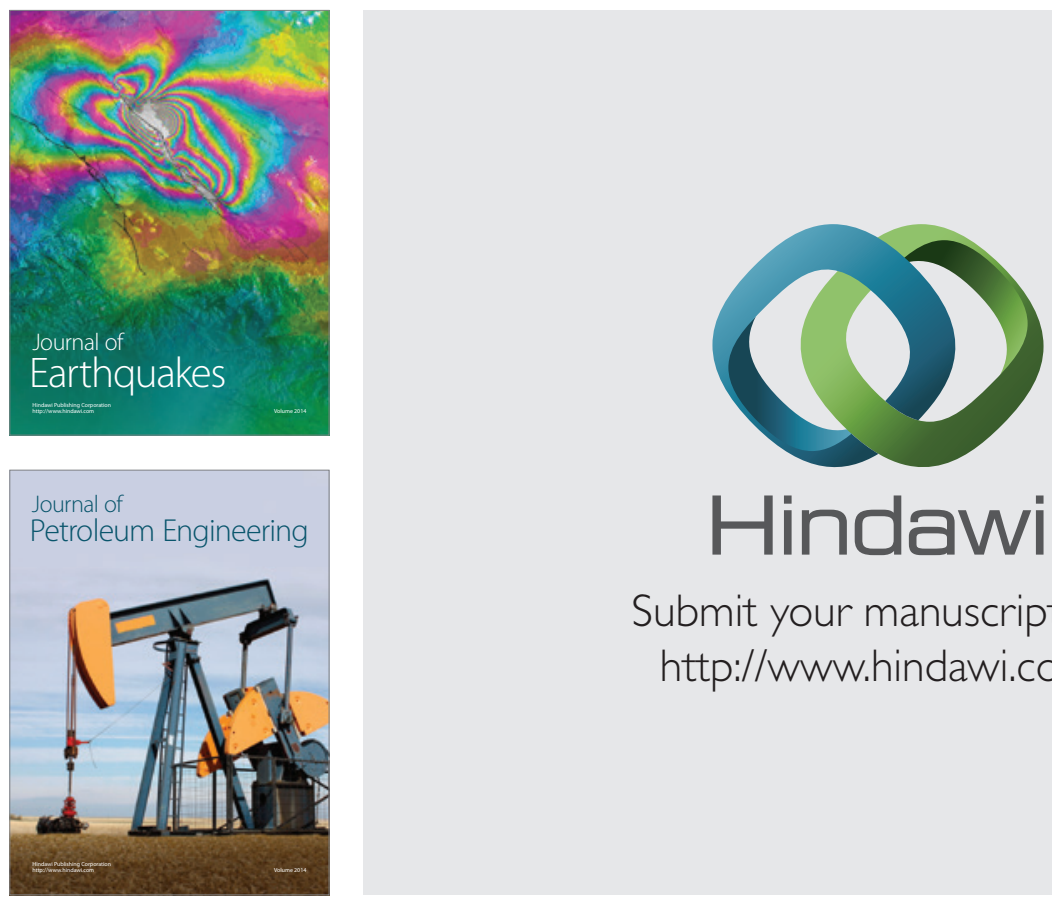

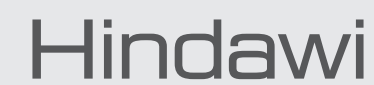

Submit your manuscripts at

http://www.hindawi.com
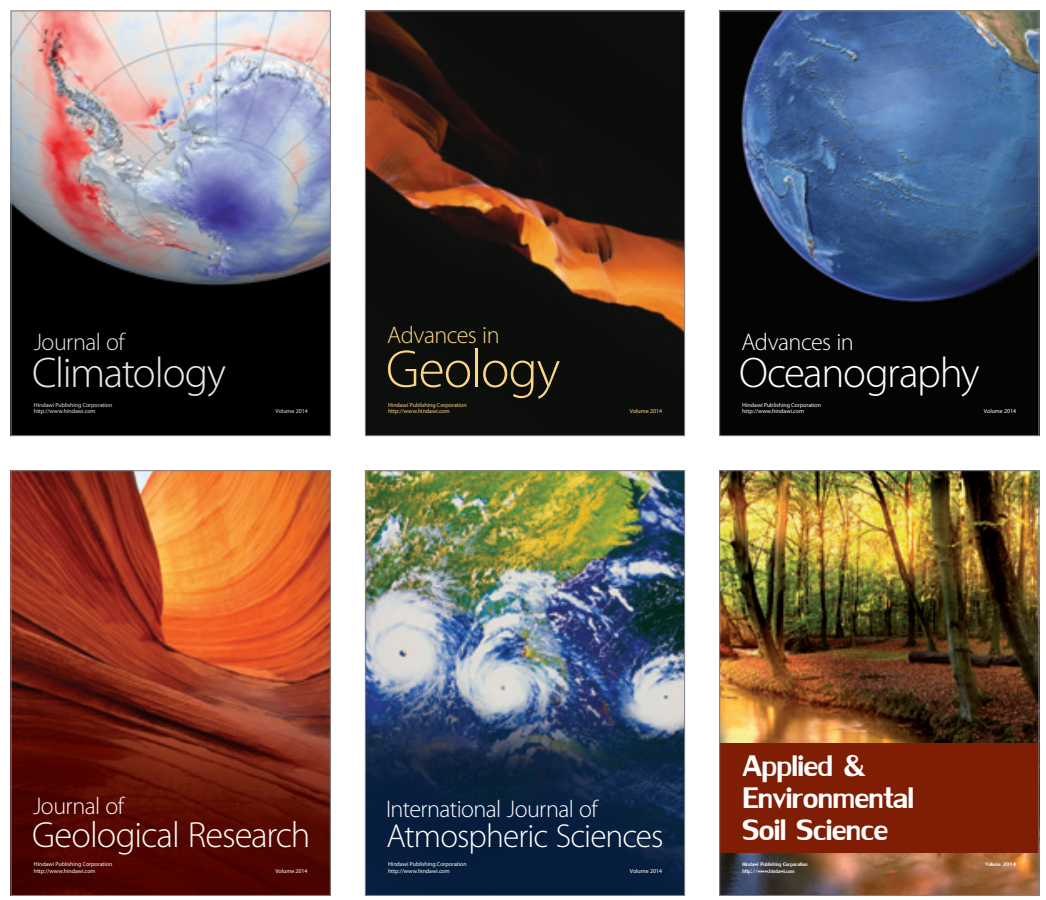
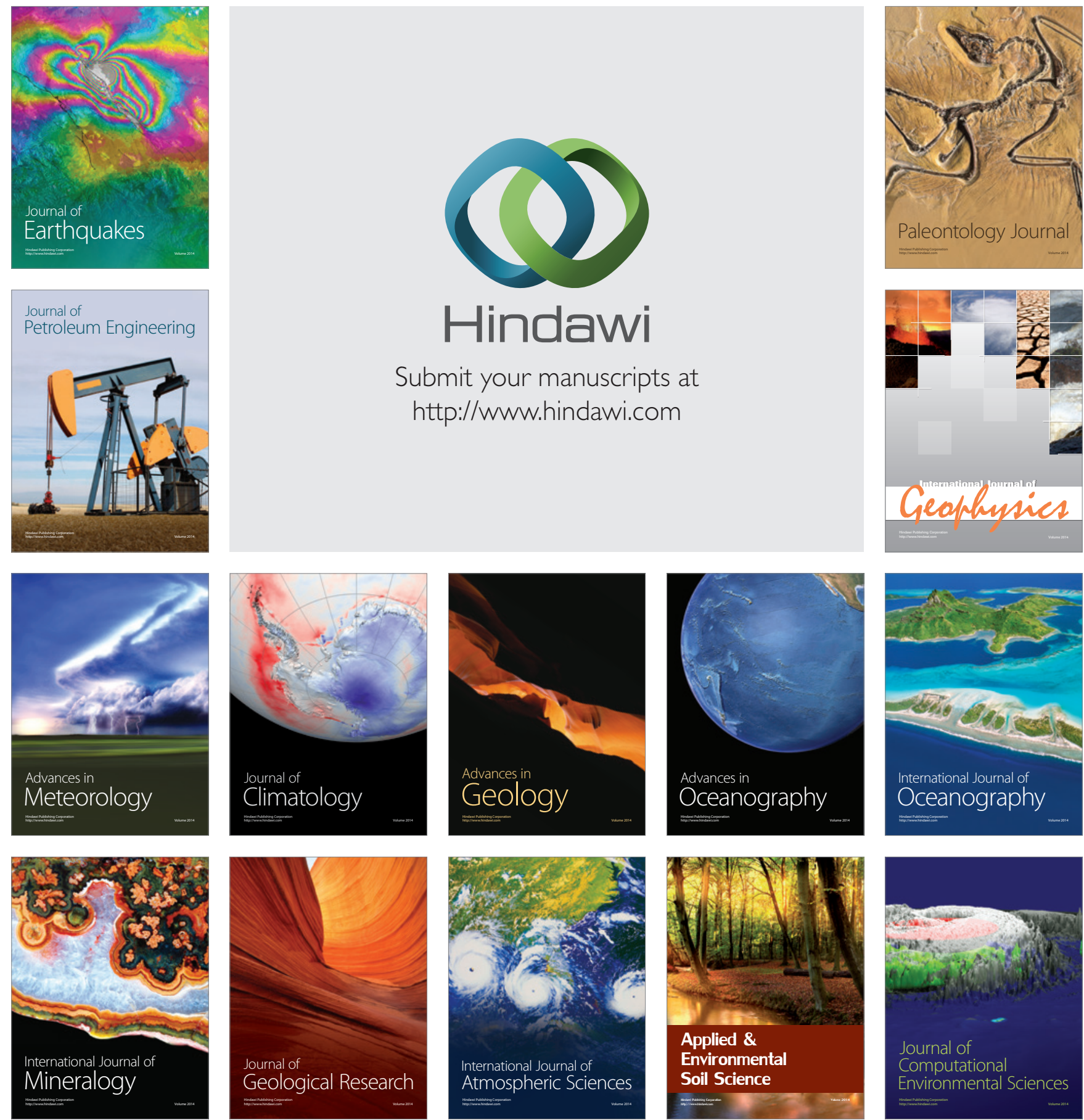